\title{
ECG Signal Compression and Classification Algorithm With Quad Level Vector for ECG Holter System
}

\author{
Hyejung Kim, Student Member, IEEE, Refet Firat Yazicioglu, Patrick Merken, \\ Chris Van Hoof, Member, IEEE, and Hoi-Jun Yoo, Fellow, IEEE
}

\begin{abstract}
An ECG signal processing method with quad level vector $(\mathrm{QLV})$ is proposed for the ECG holter system. The ECG processing consists of the compression flow and the classification flow, and the QLV is proposed for both flows to achieve better performance with low-computation complexity. The compression algorithm is performed by using ECG skeleton and the Huffman coding. Unit block size optimization, adaptive threshold adjustment, and 4-bit-wise Huffman coding methods are applied to reduce the processing cost while maintaining the signal quality. The heartbeat segmentation and the R-peak detection methods are employed for the classification algorithm. The performance is evaluated by using the Massachusetts Institute of Technology-Boston's Beth Israel Hospital Arrhythmia Database, and the noise robust test is also performed for the reliability of the algorithm. Its average compression ratio is 16.9:1 with $0.641 \%$ percentage root mean square difference value and the encoding rate is $6.4 \mathrm{kbps}$. The accuracy performance of the R-peak detection is $100 \%$ without noise and $95.63 \%$ at the worst case with -10-dB SNR noise. The overall processing cost is reduced by $45.3 \%$ with the proposed compression techniques.
\end{abstract}

Index Terms-Biomedical monitoring, biomedical signal processing, data compression, signal classification.

\section{INTRODUCTION}

$\mathbf{R}$ ECENTLY, with the increase of the interests in the healthcare, the need for the ECG holter system has been rising exponentially. The holter system records ECG signal continuously in ambulatory condition for a sizable time like several hours. The system transmits the record data to the user or the healthcare center like hospital when the alert ECG signal is detected or the recording period is finished. In order to monitor and analyze the ECG signal, the functions operated at the clinical instrument such as signal sensing and the classification, should be integrated into the light-weight, wearable holter system [1]. The most important requirements for the holter system are ultralow-energy operation for the long battery lifetime and a small footprint for wearability. The low energy consumption can be obtained by reduction of the computational complexity, the memory access, and the data transmission. In general, the highest energy consuming parts are the memory transaction blocks

Manuscript received March 20, 2009; revised July 15, 2009. First published September 22, 2009; current version published January 15, 2010.

H. Kim, R. F. Yazicioglu, P. Merken, and C. Van Hoof are with the Interuniversity Microelectronics Center, Leuven 3001, Belgium (e-mail: hyejung@imec.be; firat@imec.be; merken@imec.be; vanhoofc@imec.be).

H.-J. Yoo is with the Korea Advanced Institute of Science and Technology, Daejeon 305-701, Korea (e-mail: hjyoo@ee.kaist.ac.kr).

Digital Object Identifier 10.1109/TITB.2009.2031638 and the communication blocks integrated in the chip [2]. Zigbee and Bluetooth are usually used for the transmission channel [3]; however, those wireless communication methods consume more transmitting power than the data processing power does. Therefore, minimizing data amount by data compression is essential to reduce the total system energy consumption.

Many ECG signal compression algorithms were introduced, and they can be classified into two major groups, the lossless and the lossy algorithms [4]. The lossless algorithms such as Lempel-Ziv-Welch (LZW) [5] and Huffman [6] do not show sizable quantization error, while the compression ratio $(\mathrm{CR})$ is generally smaller than that of the lossy algorithm. The CR is typically between $2: 1$ and $4: 1$. The lossy algorithm has a comparatively higher CR, typically between 10:1 and 20:1, while it has a possibility to lose the significant information. The lossy algorithm can be classified further into two categories: the direct signal compression and the transformation compression. The direct compression techniques are based on the extraction of a subset of significant samples, such as the FAN [7], CORTES [8], AZTEC [9], and Turning Point [10] algorithms. The transformation techniques retain the coefficients of its particular features; and the signal reconstruction can be achieved by an inverse transformation process. Wavelet transform [11]-[15], Fourier transform [16], and the Karhunen-Loeve transform [17] have been introduced for the transformation compression techniques. In contrast to the direct compression techniques, the transformation techniques require heavy arithmetic calculation and large temporary memory capacity due to their large-scaleframe-based transformation operation. For the lossy compression techniques, the reduction of the reconstruction error rate is also important issue, because the error may distort diagnostic information. Moreover, the processing cost is a critical factor in the design of the holter system. The processing cost is composed of the encoding delay time, computational complexity, and the memory capacity. Thus, the tradeoff should be made between the CR, the reconstruction error, and the processing cost according to the target applications.

The feature extraction and the heartbeat classification methods have been studied, which are also essential for ECG signal processing. The feature extraction has been investigated by ECG morphology [18], [19], heartbeat interval features [18]-[20], and frequency-based features [21] methods. And the employed classification methods include linear discriminants [21], backpropagation neural networks [18], and learning vector quantization [20]. However, they are usually employed for the 


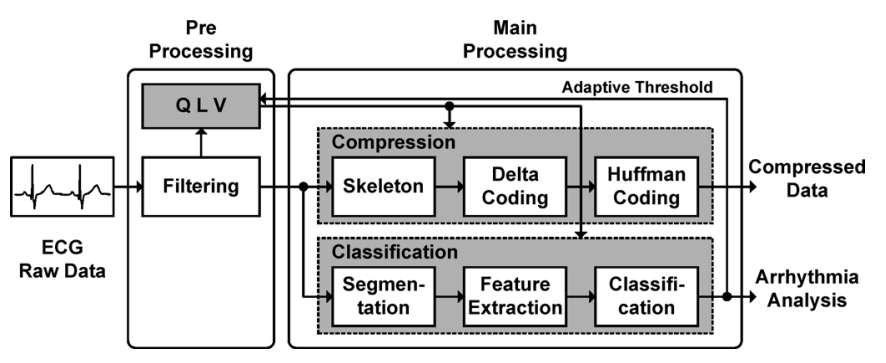

Fig. 1. Flow diagram of the proposed ECG signal processing algorithm.

clinical instruments, not holter system, where the highcomputation complexity and the large memory capacity are available. Thus, the light algorithm optimized for the holter system should be selected while maintaining the accuracy.

In this paper, the three-step compression algorithm and the ECG classification algorithm are proposed for the continuous ECG holter system. Fig. 1 shows the flow diagram of the proposed ECG signal-processing algorithm. It consists of two stages: the preprocessing stage and the main processing stage. The ECG sensing data are digitized and transmitted to the preprocessing stage. At the preprocessing stage, the filtering unit is applied to reduce the noises such as baseline wander, power line interference, and high-frequency noise. In this study, two-stage finite-impulse response (FIR) filters are implements with 10-tap filters and the programmable coefficients. By applying the programmable coefficients, the FIR filters can be used as low-pass filter or high-pass filter. The main processing stage consists of the compression flow and the classification flow. After filtering in the preprocessing stage, the quad level vector (QLV), which indicates the ECG waveform delineation and its information level, is generated for the main processing stage. The QLV support both the flows to achieve better performance with low computational complexity. The compression flow combines the lossy and the lossless algorithm, which are the skeleton, delta coding, and the Huffman coding. The classification flow extracts the features and analyzes whether the current heartbeat has abnormal arrhythmia. Moreover, the unit block size optimization for maintaining the signal quality, adaptive threshold adjustment for the algorithm reliability, and the 4-bit-wise Huffman coding methods for memory capacity reduction the computational complexity are applied.

This paper is organized as follows. The detailed explanation about the compression and the classification techniques are presented in Section II and III, respectively. In Section IV, the evaluation results are reported, and the noise injection effect is also discussed. Finally, conclusions are made in Section V.

\section{COMPRESSION FlOW}

\section{A. Skeleton of ECG Data}

The ECG signal can be divided into a crucial part and a plain part [14]. The QRS complex wave is the most important part of the cardiology system to determine arrhythmia [13]. The $\mathrm{P}$ - and T-waves also have a high level of information, and the remaining plane parts of TP segment contain less information.
Therefore, in this work, the ECG signal is classified into four different levels to preserve as much property of the information as possible. Afterward, the number of bits is assigned differently according to the level. For example, more bits are assigned to the highest level block, and fewer bits are assigned to the lower level block.

In this way, the ECG data are divided into smaller blocks and every block is encoded in real time as an independent entity. The block length is decided according to the sampling rate as (1) shows

$$
\begin{aligned}
& N_{B}=f_{\text {truncn }}\left(t_{\text {unit }} S R,\left\lfloor\log _{2}\left(\frac{S R}{c}\right)\right\rfloor\right) \\
& \text { where } f_{\text {truncn }}(x, n)=\frac{\left\lfloor x 2^{n}+0.5\right\rfloor}{2^{n}}
\end{aligned}
$$

where $N_{B}$ represents the block length, $S R$ is the ECG sampling rate, $c$ is the programmable constant between 100 and 200, $t_{\text {unit }}$ is unit block size, and $f_{\text {truncn }}$ is the rounding-truncation function with $2^{n}$. In this work, the unit block size is selected in $0.04 \mathrm{~s}$, which is the half duration of the QRS complex duration. It is a suitable period to detect the change the ECG signal precisely.

After block division, the QLV of the block is calculated. For normal ECG signals, the QRS complex part can be regarded as a typical representative signal with high standard deviation $\left(\mathrm{STD}=\sqrt{\sum_{I=0}^{N_{B}-1}\left(x_{i}-\bar{x}\right)^{2} / N_{B}}\right)$ in comparison with the plain part [14]. The complex block with high STD has more crucial information than the plain block with low STD. However, the STD requires the complex calculations such as square root $(\sqrt{ } x)$ and squaring $\left(x^{2}\right)$. Therefore, the mean deviation (MD) value is proposed to determine the QLV instead of the STD. The MD is defined as follows:

$$
\mathrm{MD}=\frac{\sum_{i=0}^{N_{B}-1}\left|x_{i}-\bar{x}\right|}{N_{B}}
$$

where $x_{i}$ is the sampled data, $\bar{x}$ is the mean value for one block, and $N_{B}$ is the block size. The MD requires the only absolute operation, thus it leads to the lower computation complexity than do the STD with the almost same results [22].

Afterward, each block is decomposed into four compression levels by comparing the MD value with the three threshold values $\left(\mathrm{TH}_{0}, \mathrm{TH}_{1}, \mathrm{TH}_{2}\right)$ as given by (3), the proposed skeleton equation.

$$
\operatorname{QLV}\left(\mathrm{CR}_{\text {block }}\right)= \begin{cases}0(8 \alpha: 1) & \text { if } \mathrm{MD}<\mathrm{TH}_{0} \\ 1(4 \alpha: 1) & \text { if } \mathrm{TH}_{0} \leq \mathrm{MD}<\mathrm{TH}_{1} \\ 2(2 \alpha: 1) & \text { if } \mathrm{TH}_{1} \leq \mathrm{MD}<\mathrm{TH}_{2} \\ 3(\alpha: 1) & \text { if } \mathrm{MD} \geq \mathrm{TH}_{2} .\end{cases}
$$

Fig. 2 shows the skeleton algorithm. The input consists of the block-wise discrete signal $\left\{x_{i}(m), i=1,2, \ldots n, m=\right.$ $\left.1,2, \ldots N_{B}\right\}$. And let $N_{B}^{l}=N_{B} / 2^{3-l}$, then the output of each block is the set $\underline{y}_{l}=\left(y_{l, 1}, y_{l, 2}, \ldots, y_{l, N_{B}^{l}}\right)^{T}$ at levels $l=0,1$, 2,3 . The final output $\left\{y_{i}\left(m_{l}\right), i=1,2, \ldots n, m_{l}=1,2, \ldots\right.$ $\left.N_{B}^{l}\right\}$ is determined by the MD value corresponding to the QLV in (3). If the CR of the block ( $\left.\mathrm{CR}_{\text {block }}\right)$ of the third level is $\alpha: 1$, 


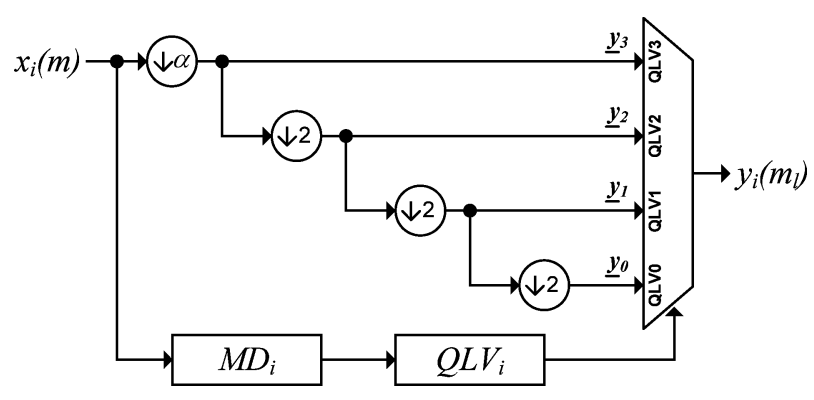

Fig. 2. Block diagram of skeleton algorithm.
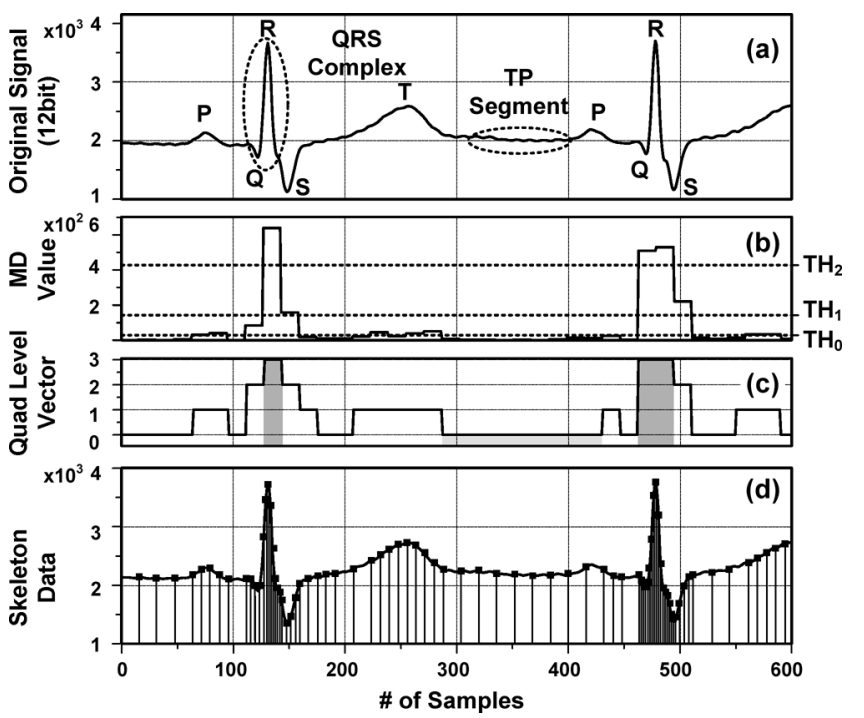

Fig. 3. Evaluated result of skeleton algorithm with MIT-BIH record 231. (a) Original ECG signal. (b) MD values. (c) QLV. (d) Skeleton results.

those of the second, first, and zeroth level are $2 \alpha: 1,4 \alpha: 1$, and $8 \alpha: 1$, respectively.

The ECG data from MIT-BIH [23] is used to verify the efficiency of the proposed skeleton algorithm. The sampling rate and the resolution of the signal are 360 samples/s and 12 bits, respectively. Fig. 3 shows the skeleton steps by the part of the MIT-BIH record 231 data.

In order to obtain the accurate QLV, it is important to choose properly the three threshold values $\left(\mathrm{TH}_{0}, \mathrm{TH}_{1}, \mathrm{TH}_{2}\right)$. Since the amplitude of ECG signal varies with the environmental conditions such as noise injection and body movements, the QLV threshold should be adaptable to deal with those variations in a real time. The threshold values are determined by the maximum $M D$ value $\left(\mathrm{MD}_{\max }\right)$ of the previous eight heartbeats, then the results is applied to the preprocessing by the feedback path for QLV adjustment of the next heartbeat. The threshold values are determined as (4).

$$
\mathrm{TH}_{l}=\frac{1}{k_{l}} \frac{\sum_{i=0}^{7} \mathrm{MD}_{\max , i}}{8}, \quad l=0,1,2
$$

where the threshold coefficients $k_{l}$ are the programmable coefficient. Fig. 4 shows the effect of the CR and the R-peak detection

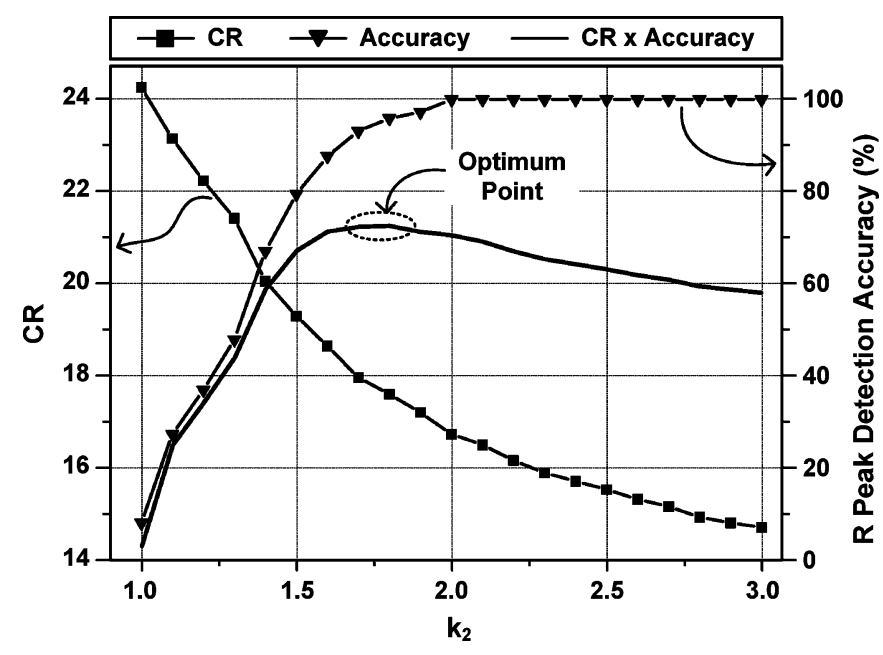

Fig. 4. Optimum point selection of the threshold coefficients $\left(k_{2}\right)$.

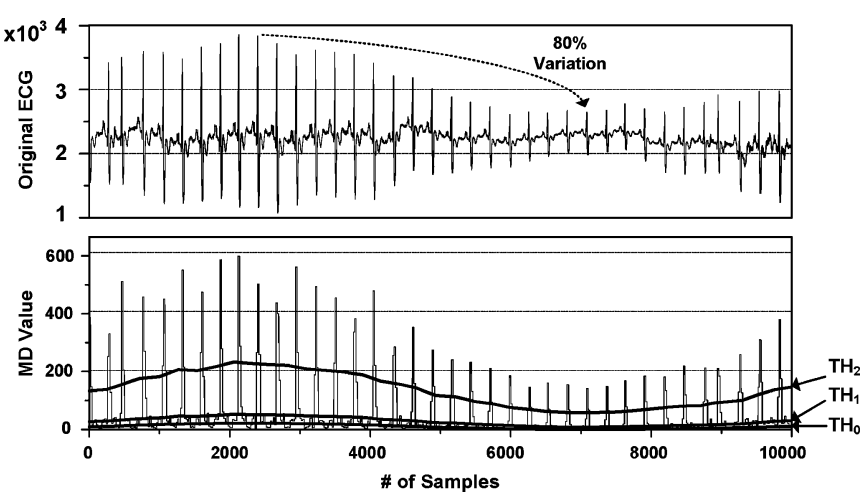

Fig. 5. Adaptive threshold value adjustment according to the ECG amplitude variation.

accuracy according to the value of the coefficient $k_{2}$. The CR increase with the decrease of the $k_{2}$. However, the lower $k_{2}$ value is very susceptible to the noise interference or the amplitude variation, and its detection accuracy is very low. On the contrary, if the coefficient values go up, the CR decreases while the accuracy improves. Therefore, there exists the optimum threshold values between the noise robustness and the accuracy, and the optimum point for the $k_{2}$ is between 1.6 and 2.0 according to the Fig. 4. Fig. 5 shows the results of the adaptive threshold value adjustment according to the amplitude variation. Although the $80 \%$ amplitude variation is applied, the threshold values can be adapted immediately to the variation after few heartbeat, thus the accurate level determination can be obtained.

The output format of the skeleton [Fig. 3(d)] consists of the signal amplitude and the sampling interval for the later decoding operation. When decoding the skeleton data, the linear interpolation method is used for the smooth reconstructed waveform with small error rate.

\section{B. Lossless Compression: Huffman Coding}

The delta coding and the lossless compression algorithm are adapted after the skeleton method. The delta coding is calculated with difference with the previous number $\left(y_{i}=x_{i}-x_{i-1}\right)$. It 
TABLE I

MODIFIED 4-Bit-Wise HuFFMAN CODING TABLE

\begin{tabular}{|c|c|c|c|c|c|c|}
\hline \multirow{2}{*}{ Group } & \multirow{2}{*}{ Value } & \multicolumn{4}{|c|}{ Number of bits } & Prefix \\
\cline { 3 - 7 } & $\begin{array}{c}\text { Prefix } \\
\text { Code }\end{array}$ & $\begin{array}{c}\text { Encoded } \\
\text { Data }\end{array}$ & $\begin{array}{c}\text { Entire } \\
\text { Code }\end{array}$ & Comments \\
(in bits) & \\
\hline \hline 0 & {$[-1,1]$} & 1 & 3 & 4 & 0 & $\begin{array}{c}0,1,-1, \text { EOB, } \\
\text { Change of QLV }\end{array}$ \\
\hline 1 & {$[-31,31]$} & 2 & 6 & 8 & 10 & \\
\hline 2 & {$[-255,255]$} & 3 & 9 & 12 & 110 & \\
\hline 3 & {$[-4095,4095]$} & 3 & 13 & 16 & 111 & \\
\hline
\end{tabular}

stores the changes instead of the absolute values to reduce the number of bit. The Huffman coding is selected because it provides minimum encoding cost when the original data has the unique distribution [6]. According to the Huffman coding scheme, the most frequently occurring values have the shortest bit code length, and the rarely occurring data have the longest bit code length. After the skeleton step, the input data have Gaussian distribution, which is more than $50 \%$ of the data are located near the zero. Thus, these high frequently occurring data can be transformed with the short length of code by the Huffman coding. Table I shows the modified 4-bit-wise Huffman coding table proposed in this paper. It divides the entire range into the four groups according to the input value to reduce the length and the number of the prefix code bits. Its output result consists of the prefix code and the encoded data. The prefix code is selected by the data probability distribution and indicates the group of the input range. The group 0 is reserved specially to notice the end of the block and the information of the QLV, while the other groups show the encoded data. The modified Huffman coding method transforms the sample-oriented format into the 4-bitword oriented stream. So, it can obtain the unified data format for the efficient memory access, although the variable sample resolution is provided. When decoding the Huffman code, the bit stream is decoded into the 4-bit-word. The first bit is picked up and compared with the Huffman table, and the original value is reconstructed from the remaining encoded data. The average CR of the Huffman coding is approximately 2:1 without the compression error rate.

\section{ECG CLASSIFICATION FLOW}

The second flow of the main processing is the classification. It consists of the segmentation, the feature extraction, and the classification stages. One heartbeat signal begins from the $\mathrm{P}$ wave and finishes at the next $\mathrm{P}$ wave of the following heartbeat. After the segmentation, the significant features of ECG, such as the $\mathrm{R}$ point, $\mathrm{RR}$ interval, amplitude of $\mathrm{R}$ point, average RR interval, QRS duration, and existence of QRS [19], should be extracted for the next classification stage. Since the proposed QLV represents the delineation of ECG signal briefly, the heartbeat segmentation and the feature extraction stages can be implemented simply by using the proposed QLV without raw data of the ECG signal. Afterward, the classification algorithm checks whether the current heartbeat has abnormal arrhythmia. The nine major disorder symptoms are chosen, such as bradcardia, tachycardia, asystole, skipped beat, R-on-T, bigeminy, trigeminy, premature ventricular contraction and atrial prema-

\begin{tabular}{|c|c|}
\hline Arrhythmia Name & Numerial Conditions \\
\hline Bradycardia & $R R_{t}>1.5 s, A R_{t}>1.2 s$ \\
\hline Tachycardia & $A R_{t}<0.5 s$ \\
\hline Asystole & $N o Q R S>1.6 s$ \\
\hline Skipped Beat & $R R_{t}>1.9 A R_{t-1}$ \\
\hline R-on-T & $R R_{t}<0.33 A R_{t-1}$ \\
\hline Bigeminy & $R R_{t-3}<0.9 A R_{t-4}, R R_{t-1}<0.9 A R_{t-4}$ \\
& $R R_{t-3}+R R_{t-2}=2 A R_{t-4}$ \\
$R R_{t-1}+R R_{t}=2 A R_{t-4}$
\end{tabular}

(a)

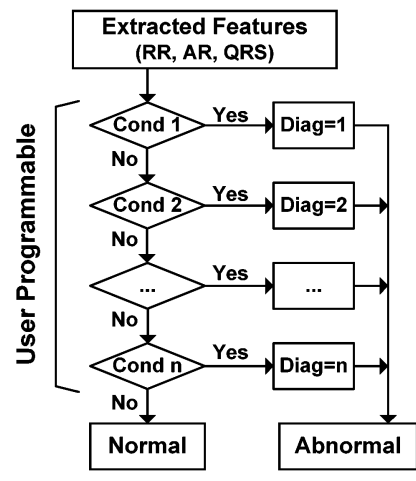

(b)

Fig. 6. (a) Selected nine major arrhythmia symptoms and their numerical conditions [24]. (b) Arrhythmia analysis flow diagram.

ture beats, and each symptom can be characterized by the simple numerical calculation [24], as shown in Fig. 6(a). Fig. 6(b) shows the overall flow diagram for the arrhythmia detection operation. When the extracted features meet the specific condition, the current heartbeat is classified as the disorder heartbeat. Otherwise, the heartbeat is regarded as normal.

The accuracy of R-peak detection is crucial for the reliable analysis in this flow, because the R-peak contains the primary data for arrhythmia analysis like RR interval [25]. During the R-peak detection operation, the QLV helps to reduce the peak searching cost. In case of the conventional system [25], the searching window would be $1 \mathrm{~s}$ same as the one heartbeat duration. In this proposed system, searching for QLV array is performed first. Then the only selected searching window, 40$80 \mathrm{~ms}$, is applied to find the real peak. Although this searching method has $1 \%$ memory capacity overhead for the QLV array, the number of memory access time is reduced by $90 \%$. Fig. 7 shows the successful R-peak detection results, using the MIT$\mathrm{BIH}$ record 100 with serious noise injection $(\mathrm{SNR}=-10 \mathrm{~dB})$ and the selected search window. By using the QLV, only $8 \%$ search window is enough for the investigation compared to the entire range, as shown in Fig. 7(b). Only one fault negative result is detected as R-peak due to the steep noise denoted by $x$ near the 3800 point of Fig. 7(a), but more preprocessing like filtering and the postprocessing can correct the fault detection. 


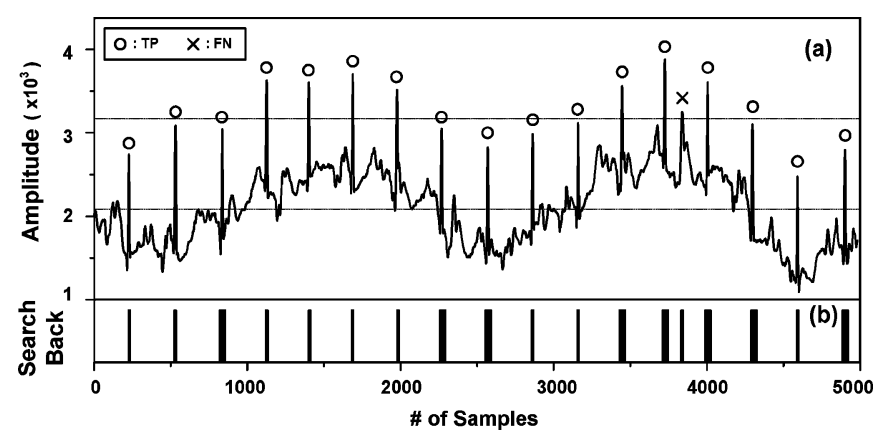

Fig. 7. (a) R-peak detection results. (b) Searching window in record 100 with noise injection $(\mathrm{SNR}=-10 \mathrm{~dB})$.

\section{EVALUATION RESULtS}

The MIT-BIH Arrhythmia Database is used to evaluate the performance [23]. The sampling rate and the resolution are 360 samples/s and 12 bits, respectively. In addition, the Gaussian white noise source is injected from -10 to $20 \mathrm{~dB}$ SNR for the noise stress test. The test vector is produced by injection of the noise source [26] with MIT-BIH record 100 [23]. The performances of compression coding are evaluated by the CR and the compression error rate of the percentage root mean square difference (PRD). And the R-peak detection performance is evaluated by the sensitivity $(\mathrm{Se})$ and the positive predictivity $(+P)$.

\section{A. ECG Compression Coding Performance}

The overall CR is calculated by the product of the two CRs, the skeleton $\left(\mathrm{CR}_{\mathrm{sk}}\right)$ and the Huffman coding $\left(\mathrm{CR}_{\mathrm{hf}}\right)$, and it is given in (5) as:

$$
\begin{aligned}
\mathrm{CR}_{\text {overall }}= & \mathrm{CR}_{\mathrm{sk}} \times \mathrm{CR}_{\mathrm{hf}}=\sum_{l=0}^{3}\left(\mathrm{CR}_{\mathrm{sk}, l} \times P_{\mathrm{sk}, l}\right) \\
& \times \sum_{s=0}^{3}\left(\mathrm{CR}_{\mathrm{hf}, g} \times P_{\mathrm{hf}, g}\right)
\end{aligned}
$$

where $l$ is the level of the skeleton, $g$ is the group of the Huffman coding, and $P$ represents the distributed percentage. According to the evaluation results of the MIT-BIH database, using 231 record, the subblock $\mathrm{CR}$ of skeleton is defined as $\mathrm{CR}_{\mathrm{sk}, 0}: \mathrm{CR}_{\mathrm{sk}, 1}: \mathrm{CR}_{\mathrm{sk}, 2}: \mathrm{CR}_{\mathrm{sk}, 3}=1 / 16: 1 / 8: 1 / 4: 1 / 2$, when let $\alpha$ be 2 in formula (2). And its percentages are $P_{\mathrm{sk}, 0}: P_{\mathrm{sk}, 1}: P_{\mathrm{sk}, 2}$ : $P_{\mathrm{sk}, 3}=60: 25: 10: 5$, so the $\mathrm{CR}_{\mathrm{sk}}$ is 8.4:1. If $\alpha$ is bigger than 2, the CR increases, of course. And the subblock CR of Huffman coding is defined as $\mathrm{CR}_{\mathrm{hf}, 0}: \mathrm{CR}_{\mathrm{hf}, 1}: \mathrm{CR}_{\mathrm{hf}, 2}: \mathrm{CR}_{\mathrm{hf}, 3}=4 / 12$ : $8 / 12: 12 / 12: 16 / 12$, according to the proposed Huffman coding table. The percentage distribution are $P_{\mathrm{hf}, 0}: P_{\mathrm{hf}, 1}: P_{\mathrm{hf}, 2}: P_{\mathrm{hf}, 3}=$ 31:52:17:0, so the $\mathrm{CR}_{\mathrm{hf}}$ is $1.88: 1$. Therefore, the overall $\mathrm{CR}$ (CR overall) would be 15.8:1 from (5).

The Fig. 8(a) and (b) shows the original ECG signal and the reconstructed results, respectively. The result shows that high-quality signal is reconstructed with small error rate. Even though the maximum peak error is $0.85 \%$, the most of samples shows $<0.1 \%$ error, as shown in Fig. 8(c). The PRD is usually

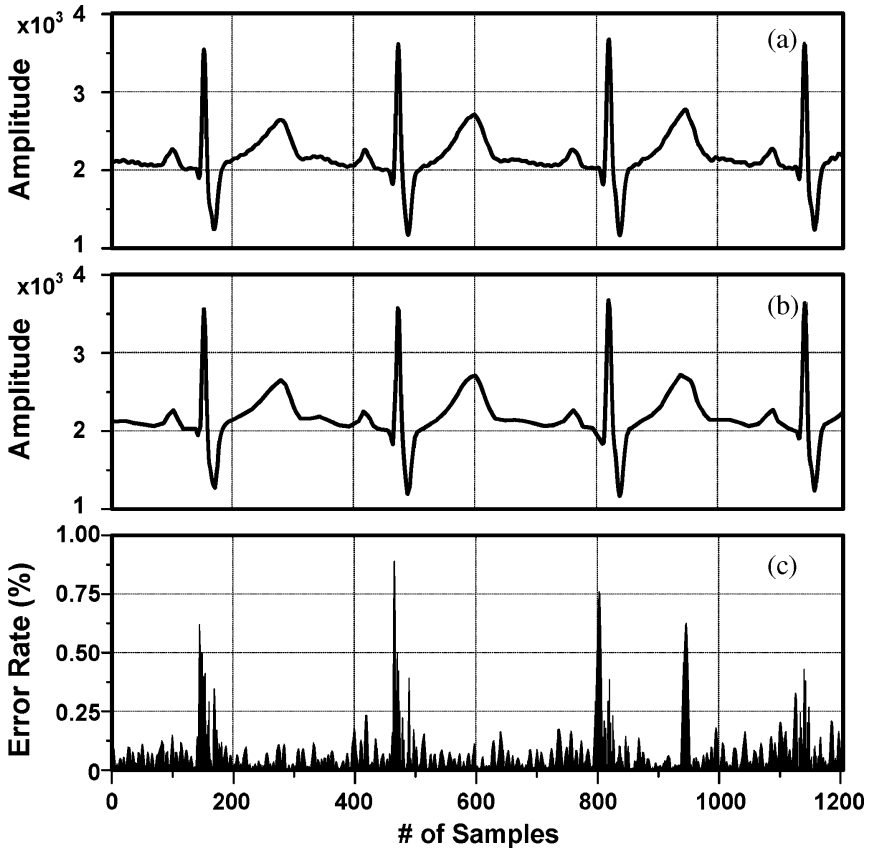

Fig. 8. (a) Original ECG signal of record 231. (b) Reconstructed ECG signals. (c) Reconstructed error rate between original and reconstructed signal.

used to quantify the performance quality of the compression algorithm [27]. The PRD indicates the error between the original ECG samples and the reconstructed data, and is defined as

$$
\operatorname{PRD}(\%)=\sqrt{\frac{\sum_{i=1}^{n}\left(x_{i}-\tilde{x}_{i}\right)^{2}}{\sum_{i=1}^{n} x_{i}^{2}}} \times 100
$$

where $n$ is the number of samples, $x_{i}$ and $\tilde{x}_{i}$ are the original data and the reconstructed data, respectively. The PRD of the Huffman coding is conserved because it is the lossless compression [6].

The CR and PRD have the close relationship in the lossy compression algorithm. In general, the CR goes higher with the higher lossy level, while the error rate goes up. The final goal of the proposed compression algorithm is to keep the PRD value smaller than that of the conventional methods [4]-[17] while maintaining the similar CR. Fig. 9(a) shows the evaluation results of the PRD as a function of CR for each record from the MIT-BIH database. The results show that all the methods have high correlation between the $\mathrm{CR}$ and the PRD. The average PRD value of the evaluation in Fig. 9(a) is 0.641\% at 16.9:1 CR.

The comparison results with the conventional methods, such as wavelet based the embedded zero-tree wavelet ECG coder [5], set partitioning in hierarchical trees (SPIHT) ECG coder [16], and the modified SPIHT ECG coder [15], are also given in Fig. 9(b). Their results show that the proposed method has better PRD performance than other conventional method in the CR range of 4-20, especially at the high CR.

The quality score $(\mathrm{QS}=\mathrm{CR} / \mathrm{PRD})$ was proposed to quantify the overall performance of the compression algorithm, considering both the CR and the error rate [27]. A high score represents a good compression performance. This work shows the $\mathrm{QS}=29.36$ when $\mathrm{CR}=16.9: 1$ and $\mathrm{PRD}=0.641 \%$. Table II 

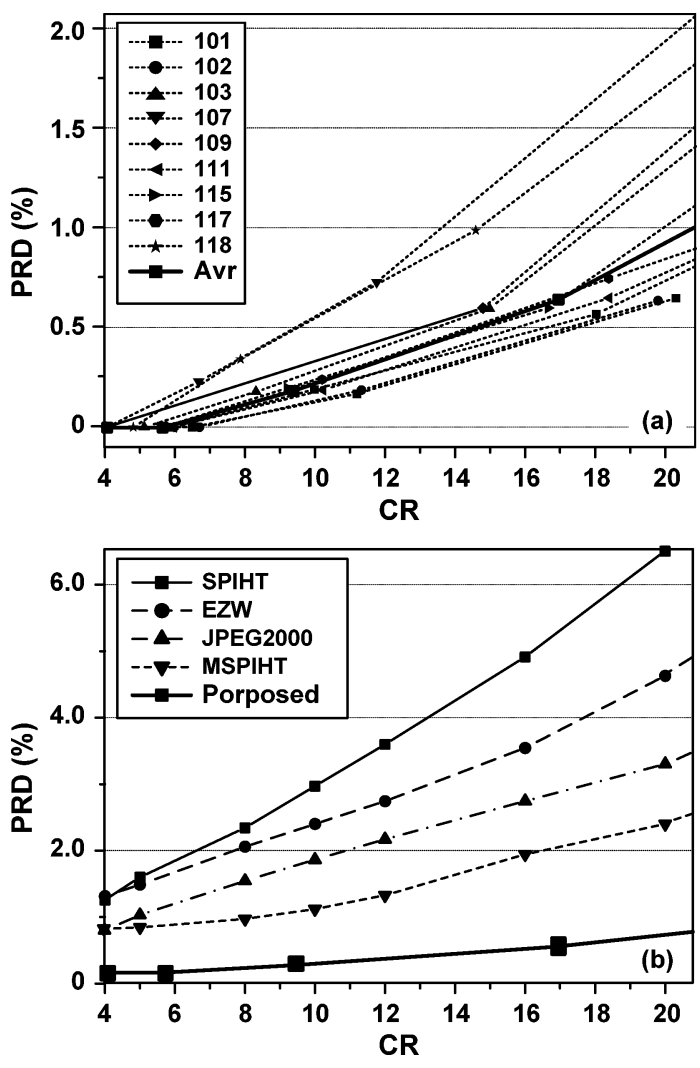

Fig. 9. (a) PRD versus CR of selected records from the MIT-BIH database. (b) Comparison results of performance of the proposed algorithm with the conventional algorithms.

TABLE II

COMPARISON RESUlTS OF QUALITY SCORE OF THE PROPOSED ALGORITHM WITH THE CONVENTIONAL ALGORITHMS

\begin{tabular}{|c|c||c|c|c|}
\hline \multicolumn{2}{|c||}{ Algorithm } & CR & PRD (\%) & QS \\
\hline \hline Hilton [11] & Wavelet (EZW) & $8: 1$ & 2.6 & 3.07 \\
\hline Djohan [12] & Wavelet (DSW) & $8: 1$ & 3.9 & 2.05 \\
\hline Manikandan [17] & DFT w/ DSI & $8: 1$ & 1.553 & 5.15 \\
\hline Kim [14] & WLDECG w/STD & $15: 1$ & 1.067 & 14.06 \\
\hline Fira [28] & Skeleton, LZW & $18.27: 1$ & 1.17 & 15.61 \\
\hline This Work & $\begin{array}{c}\text { QLV-Skeleton, } \\
\text { Huffman }\end{array}$ & $16.9: 1$ & 0.641 & 29.36 \\
\hline
\end{tabular}

compares the QS of the proposed method with those of the conventional methods. The first three methods have poor CR and the PRD performance. The Kim's method [14] had the similar CR but showed higher PRD, because the transformation compression method produces some reconstruction distortions. The Fira's method [27] also used the two-step compression algorithm, skeleton, and the LZW. Although it has high CR, however, its PRD is also higher because the peak-picking skeleton algorithm is not accurate than this work. With respect to computation requirements, the encoding rate is also evaluated. The encoding rates of [11], [12], [27] are not reported, and only the encoding rate of Kim's [13] is compared with this method. The encoding rate is normalized with 12-bit data processing, and it is $6.4 \mathrm{kbps}$ for this work, which is much higher than that of $2.56 \mathrm{kbps}$ for Kim's.
TABLE III

R Peak-Detection Performance With the Noise InJection

\begin{tabular}{|c||c|c|c|c|c|c|c|c|}
\hline SNR (dB) & $\mathbf{- 1 0}$ & $\mathbf{- 5}$ & $\mathbf{0}$ & $\mathbf{5}$ & $\mathbf{1 0}$ & $\mathbf{1 5}$ & $\mathbf{2 0}$ & $\begin{array}{c}\text { No } \\
\text { Noise }\end{array}$ \\
\hline \hline Sensitivity (\%) & 95.63 & 97.38 & 98.25 & 100 & 100 & 100 & 100 & 100 \\
\hline $\begin{array}{c}\text { Positive (\%) } \\
\text { Productivity (\%) }\end{array}$ & 97.04 & 99.70 & 100 & 100 & 100 & 100 & 100 & 100 \\
\hline
\end{tabular}

TABLE IV

COMPARISON RESUlT OF R-PEAK DETECTION ACCURACY

\begin{tabular}{|c||c|c|}
\hline & $\boldsymbol{S e}$ & $+\boldsymbol{P}$ \\
\hline \hline Benitez [29] & $99.81 \%$ & $99.83 \%$ \\
\hline Benitez [30] & $99.94 \%$ & $99.93 \%$ \\
\hline Hickey [31] & $98.5 \%$ & $98.4 \%$ \\
\hline Arzeno [26] & $99.68 \%$ & $99.63 \%$ \\
\hline This Work & $100 \%$ & $100 \%$ \\
\hline
\end{tabular}

\section{B. R-Peak Detection Performance}

The performance of the classification can be represented by the sensitivity $(\mathrm{Se})$ and the positive predictivity $(+P)$. The $\mathrm{Se}$ and $+P$ are defined as follows:

$$
\begin{aligned}
\text { Sensitivity }(\mathrm{Se}) & =\frac{\mathrm{TP}}{\mathrm{TP}+\mathrm{FN}} \\
\text { Positive P redictivity }(+P) & =\frac{\mathrm{TP}}{\mathrm{TP}+\mathrm{FP}}
\end{aligned}
$$

where false positive (FP) is the number of false beat detection, true positive (TP) is the total number of correct R-peak detection by the algorithm, and false negative (FN) is the number of the failures to detect the true beat. The proposed method has good sensitivity and positive predictivity, $\mathrm{Se}=100 \%$ and $+P=100 \%$. For the noise robustness test, the signal with seven different level of injected noise was used from -10 to $20 \mathrm{~dB}$ SNR. Table III shows the sensitivity and the positive predictivity results in proportion to the noise level. According to the analysis results, the R-peak detection accuracy is very high at the low-level noise condition, but some false detection occurs at the high-level noise. The Se and $+P$ are $95.63 \%$ and $97.04 \%$, respectively, at $-10 \mathrm{~dB}$ SNR (worst case). Table IV shows the R-peak detection accuracy performance and the comparison with the previous works [25], [28]-[30], and we can see that the performance of this work is better than the others.

\section{Processing Cost}

The test vector for one minute ECG signal is used to evaluate the processing cost required in the skeleton, the Huffman coding, and the classification flow. The evaluation environment is $1 \mathrm{MHz}$ operating frequency with $8 \mathrm{kB}$ RAM running MSP 430 microcontroller. The processing cost is calculated based on the processing time, the power consumption, the memory capacity, and the number of the memory access. They can be merged to the energy consumption index, as shown in Fig. 10. Moreover, the graph shows that the overall energy can be reduced by using the proposed compression technique. Although the proposed compression technique needs memory capacity and preprocessing overhead, it can reduce the postprocessing time. As a result, 


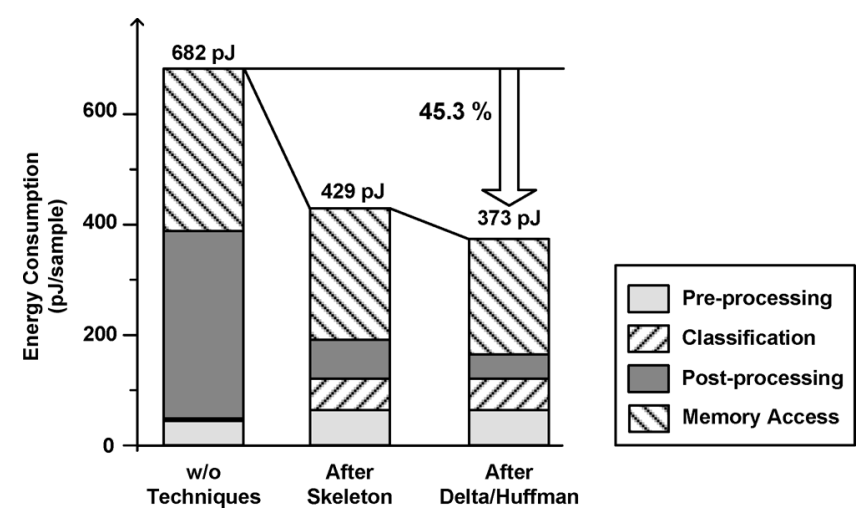

Fig. 10. Overall energy reduction with proposed compression technique.

the overall energy consumption is reduced by $45.3 \%$, and the final energy consumption is $373 \mathrm{pJ} / \mathrm{sample}$.

\section{CONCLUSION}

An ECG signal processing method consisting of the three-step compression flow and the classification flow is proposed for the Holter system. The proposed QLV delineate the ECG signal, and it supports both the flows to achieve better performance with low computation complexity. By applying the QLV, the overall CR improves while maintaining the small compression error rate, and high-accuracy performance can achieve at the segmentation and the R-peak detection stage. The performance is evaluated by using the MIT/BIH Arrhythmia Database, and the noise robust test is also performed for the reliability of the algorithm. The average CR is 16.9:1 with $0.641 \%$ PRD value, and the encoding rate is $6.4 \mathrm{kbps}$. The accuracy performance of the R-peak detection is $100 \%$ without noise and $97.5 \%$ at the worst case with $-10 \mathrm{~dB}$ SNR noise. The overall energy consumption is $373 \mathrm{pJ} / \mathrm{sample}$ when all functions algorithms are performed and is achieved $45.3 \%$ reduction.

\section{REFERENCES}

[1] D. Jabaudon, J. Sztajzel, K. Sievert, T. Landis, and R. Sztajzel, "Usefulness of ambulatory 7-day ECG monitoring for the detection of atrial fibrillation and flutter after acute stroke and transient ischemic attack," Stroke, J. Amer. Heart Assoc., vol. 35, pp. 1647-1651, May 2004.

[2] H. Kim, S. Choi, and H.-J. Yoo, "A low power 16-bit RISC with lossless compression accelerator for body sensor network system," in Proc. IEEE ASSCC, Nov. 2006, pp. 207-210.

[3] S.-J. Song, N. Cho, S. Kim, J. Yoo, and H.-J. Yoo, "A 2 Mb/s wideband pulse transceiver with direct-coupled interface for human body communications," in Proc. IEEE ISSCC, Feb. 2006, pp. 2278-2287.

[4] Y. Zigel, A. Cohen, and A. Katz, "The weighted diagnostic distortion (WDD) measure for ECG signal compression," IEEE Trans. Biomed. Eng., vol. 47, no. 11, pp. 1422-1430, Nov. 2000.

[5] T. A. Welch, "A technique for high-performance data compression," Computer, vol. 17, no. 6, pp. 8-19, Jun. 1984.

[6] Health Informatics. Standard Communication Protocol. Computerassisted Electrocardiography, British-Adopted European Standard BS EN 1064, 2005.

[7] D. A. Dipersio and R. C. Barr, "Evaluation of the fan method of adaptvie sampling on human electrocardiograms," Med. Biol. Eng. Comput., vol. 23, pp. 401-410, Sep. 1985.

[8] J. P. Abenstein and W. J. Tompkins, "A new data reduction algorithm for real time ECG analysis," IEEE Trans. Biomed. Eng., vol. BME-29, no. 1, pp. 43-48, Apr. 1982.
[9] J. R. Cox, F. M. Nolle, H. A. Fozzard, and G. C. Oliver, "AZTEC, a preprocessing program for real time ECG rhythm analysis," IEEE Trans. Biomed. Eng., vol. BME-15, no. 4, pp. 128-129, Apr. 1968.

[10] W. C. Mueller, "Arrhythmia detection program for an ambulatory ECG monitor," Biomed. Sci. Instrum., vol. 14, pp. 81-85, 1978.

[11] M. L. Hilton, "Wavelet and wavelet packet compression of electrocardiograms," IEEE Trans. Biomed. Eng., vol. 44, no. 5, pp. 394-402, May 1997.

[12] A. Djohan, T. Q. Nguyen, and W. J. Tompkins, "ECG compression using discrete symmetric wavelet transform," in Proc. IEEE EMBC, 1995, vol. 1, pp. $167-168$.

[13] R. S. H. Istepanian and A. A. Petrosian, "Optimal zonal wavelet-based ECG data compression for mobile telecardiology system," IEEE Trans. Inf. Technol. Biomed., vol. 4, no. 3, pp. 200-211, Sep. 2000.

[14] B. S. Kim, S. K. Yoo, and M. H. Lee, "Wavelet-based low-delay ECG compression algorithm for continuous ECG transmission," IEEE Trans. Inf. Technol. Biomed., vol. 10, no. 1, pp. 77-83, Jan. 2006.

[15] S.-C. Tai, C.-C. Sun, and W.-C. Yan, "A 2-D ECG compression method based on wavelet transform and modified SPIHT," IEEE Trans. Biomed. Eng., vol. 52, no. 6, pp. 999-1008, Jun. 2005.

[16] M. S. Manikandan and S. Dandapat, "ECG signal compression using discrete sinc interpolation," in Proc. IEEE ICISIP, Dec. 2005, pp. 14-19.

[17] S. Olmos, M. MillAn, J. Garcia, and P. Laguna, "ECG data compression with the Karhunen-Loeve transform," Comput. Cardiol., vol. 8-11, pp. 253-256, Sep. 1996

[18] Y. H. Hu, W. J. Tompkins, J. L. Urrusti, and V. X. Afonso, "Applications of artificial neural networks for ECG signal detection and classification," J. Electrocardiol., vol. 26, pp. 66-73, 1993.

[19] P. de Chazal, S. Palreddy, and W. J. Tompkins, "Automatic classification of hearbeats using ECG morphology and heartbeat interval features," IEEE Trans. Biomed. Eng., vol. 51, no. 7, pp. 1196-1206, Jul. 2004.

[20] Y. H. Hu, S. Palreddy, and W. J. Tompkins, "A patient-adaptable ECG beat classifier using a mixture of experts approach," IEEE Trans. Biomed. Eng., vol. 44, no. 9, pp. 891-900, Sep. 1997.

[21] L. Senhadji, G. Carrault, J. J. Bellanger, and G. Passariello, "Comparing wavelet transforms for recognizing cardiac patterns," IEEE Eng. Med. Biol. Mag., vol. 14, no. 2, pp. 167-173, Mar./Apr. 1995.

[22] H. Kim, Y. Kim, and H.-J. Yoo, "A low cost quadratic level ECG compression algorithm and its hardware optimization for body sensor network system," in Proc. IEEE EMBC, Aug. 2008, pp. 5490-5493.

[23] (1979). [Online]. Available: http://www.physionet.org/physiobank/ database $/ \mathrm{mitdb} /$

[24] D. C. Reddy, Biomedical Signal Processing-Principles and Techniques. New York: McGraw-Hill, 2005.

[25] N. M. Arzeno, Z.-D. Deng, and C.-S. Poon, "Analysis of first-derivative based QRS detection algorithms," IEEE Trans. Biomed. Eng., vol. 55, no. 2 , pp. $478-484$, Feb. 2008

[26] (1992). [Online]. Available: http://www.physionet.org/physiobank/ database/nstdb/

[27] C. M. Fira and L. Goras, "An ECG signals compression method and its validation using NNs," IEEE Trans. Biomed. Eng., vol. 55, no. 4, pp. 1319-1326, Apr. 2008.

[28] D. S. Benitez, P. A. Gaydecki, A. Zaidi, and A. P. Fitzpatrick, "A new QRS detection algorithm based on the Hilbert transform," Comput. Cardiol., vol. 27, pp. 379-382, 2000.

[29] D. S. Benitez, P. A. Gaydecki, A. Zaidi, and A. P. Fitzpatrick, "The use of the Hilbert transform in ECG signal analysis," Comput. Biol. Med., vol. 31, pp. 399-406, 2001.

[30] B. Hickey et al., "Non-episode-dependent assessment of paroxysmal artial fibrillation through measurement of RR interval dynamics and atrial premature contractions," Ann. Biomed. Eng., vol. 32, no. 5, pp. 677-687, 2004.

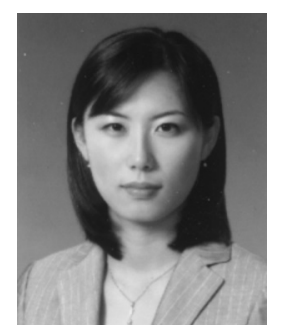

Hyejung Kim (S'04) received the B.S. and M.S. and Ph.D. degrees from the Korea Advanced Institute of Science and Technology, Daejeon, Korea, in 2004. 2006, and 2009, respectively.

She is currently a Postdoctoral Researcher with the Interuniversity Microelectronics Center, Leuven, Belgium. Her research interests include design and implementation of low-energy biomedical signal processors for body sensor network application. 


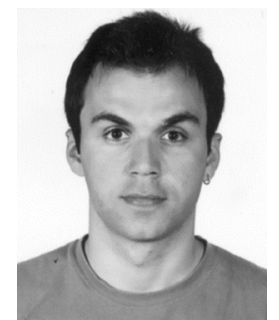

Refet Firat Yazicioglu received the B.S. and M.S. degrees in electrical and electronics engineering from the Middle East Technical University (METU), Ankara, Turkey, in 2001 and 2003, respectively, and the $\mathrm{Ph} . \mathrm{D}$. degree in electronics Engineering from the Katholieke Universiteit Leuven, Leuven, Belgium, in 2008.

From 2001 to 2003, he was a Research Assistant with the METU, where he was engaged in research on fabrication of surface micromachined capacitive accelerometers and their readout circuits. From 2003 to 2008 , his Ph.D. studies was engaged in research on the design of low-power and low-noise readout circuits for portable biopotential acquisition systems, $\mathrm{He}$ is currently a Researcher with the Interuniversity Microelectronics Center, Leuven, Belgium. His research interests include design of ultralow-power and high-performance circuits for biomedical applications.

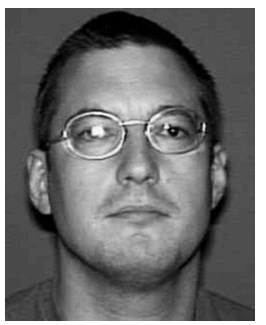

Patrick Merken received the Engineer degree in electrical engineering from the Royal Military Academy, Polytechnical Division, Brussels, Belgium, in 1988, the Master of Engineering in material science from the University of Leuven, Leuven, Belgium, in 1996, and the Ph.D. degree in electrical engineering from the University of Leuven.

During his Ph.D. studies, he was engaged in research on hybrid integration of III-V type semiconductors and silicon CMOS VLSI circuits. He is currently a Professor with the Royal Military Academy, Brussels, Belgium. He is also with the Interuniversity Microelectronics Center (IMEC), Leuven, Belgium, where he is engaged in research on design and implementation of low-noise, low-power sensor readout electronics, and cryogenic circuits.

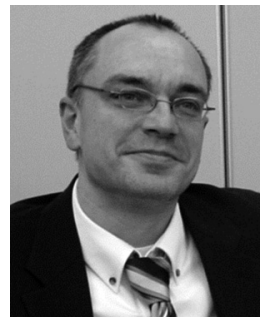

Chris Van Hoof (M'87) received the Ph.D. in electrical engineering from the University of Leuven, Leuven, Belgium, in collaboration with the Interuniversity Microelectronics Center (IMEC), Leuven, Belgium, in 1992.

$\mathrm{He}$ is currently the Integrated Systems Director and the Program Director at the IMEC, where he became successively the Head of the Detector Systems Group (in 1998), the Director of the Microsystems and Integrated Systems Department (in 2002), and the Program Director (in 2007). His research interests include the application of advanced packaging and interconnect technology (2-D and 3-D integration, RF integration) and ultralow power design technology for the creation of integrated systems, ultralow power wireless autonomous sensor systems, and smart implantable devices. His work has resulted in flight hardware for two cornerstone European Space Agency missions, and he is a laureate of the Belgian Royal Academy of Sciences. Since 2000, he has been a Professor at the University of Leuven (Katholieke Universiteit Leuven), where he is the promoter of eight doctoral theses.

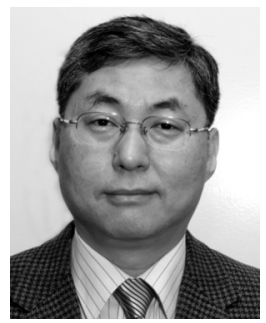

Hoi-Jun Yoo (F'08) graduated from the Electronic Department, Seoul National University, Seoul, Korea, in 1983, and received the M.S. and Ph.D. degrees from the Department of Electrical Engineering, Korea Advanced Institute of Science and Technology (KAIST), Daejeon, Korea, in 1985 and 1988, respectively.

From 1988 to 1990, he was with the Bell Communications Research, Red Bank, NJ. In 1991, he became a manager of the Dynamic Random Access Memory (DRAM) Design Group, Hyundai Electronics and designed a family of fast-1 M DRAMs to $256 \mathrm{M}$ synchronous DRAMs. In 1998, he joined the Faculty of the Department of Electrical Engineering, KAIST, where he is currently a Full Professor. From 2003 to 2005, he was the Full Time Advisor to Minister of the Korea Ministry of Information and Communication and National Project Manager for System on a Chip (SoC) and Computer. In 2007, he founded System Design Innovation and Application Research Center, KAIST, where he was engaged in research on SoCs for intelligent robots, wearable computers, and biosystems. His research interests include high-speed and low-power network on chips, 3-D graphics, body area networks, biomedical devices and circuits, and memory circuits and systems. 